\title{
OUTCOME OF VAGINAL VERSUS ABDOMINAL ROUTE OF HYSTERECTOMY
}

\author{
DR. MUDASIRA HABIB, MBBS \\ NISHTAR HOSPITAL, MULTAN, PAKISTAN. \\ DR. FAKHAR IQBAL, MBBS \\ NISHTAR HOSPITAL, MULTAN, PAKISTAN. \\ DR. HASSAN SAIF KHAN, MBBS \\ NISHTAR HOSPITAL, MULTAN, PAKISTAN.
}

\begin{abstract}
Background; Hysterectomy is "Surgical removal of all or part of uterus". Abdominal and vaginal route of hysterectomies are both predominant operative techniques being employed by the gynecologists all the world for various uterine conditions. Indications to select any particular technique in any of the hospital setting might not be optimally defined. This study was planned to evaluate particular route of hysterectomy (vaginal hysterectomy and abdominal hysterectomy) as there was no such study available which could document the data of our local population so this study was planned to be conducted to determine burden of problem and deficiencies for clinicians to opt better treatment options among targeted population. The results of this study will not only add in national data but will also be comparable with other international studies. Objective; To determine outcome of vaginal versus abdominal route of hysterectomy at a tertiary care hospital. Material and methods: A total of 240 women meeting inclusion and exclusion criteria of this study were registered in this study. Informed consent was taken from each patient. All the relevant data were recorded on pre-designed proforma. Data were entered and analyzed using SPSS-17. Results: A total of 240 subjects fulfilling the inclusion and exclusion criteria were registered in this study for abdominal hysterectomy and vaginal hysterectomy. Out of these 240 cases, 79 $(32.9 \%)$ had undergone vaginal hysterectomy and $161(67.1 \%)$ underwent through abdominal hysterectomy. Mean age of the study cases was $42.72 \pm 5.18$ years (minimum age was 31 years while maximum age was up to 50 years). Mean parity of the study cases was $5.67 \pm 2.08$ (minimum para 1 and maximum para 10). Pyrexia was seen in $90(37.5 \%)$ of the study cases, of these $27(30 \%)$ had undergone through vaginal hysterectomy and 63 (70\%) underwent abdominal hysterectomy. Minimum duration of the surgery was 45 minutes and maximum duration of the surgery was 90 minutes, mean duration of the surgery was $69.75 \pm 14.92$ minutes. Minimum Hospital stay was 3 days ranging to maximum hospital stay being 10 days, mean hospital stay in these study cases was observed to be $4.42 \pm 1.65$ days. Conclusion; Vaginal route of hysterectomy is associated with lesser postoperative complications in terms short duration surgery, significantly short hospital stays and lower rates of postoperative pyrexia than that of abdominal route of hysterectomy.
\end{abstract}

Keywords; Abdominal Hysterectomy, Vaginal hysterectomy, Uterovaginal prolapse.

DOI: $10.7176 / \mathrm{JMPB} / 55-05$

Publication date:May $31^{\text {st }} 2019$

\section{Introduction;}

Hysterectomy is "Surgical removal of all or part of uterus". The first abdominal hysterectomy was performed by Charles Clay in Manchester in 1843. A total of 575, 000 hysterectomies were done in USA in year $2005^{1}$. Nine out of every 10 hysterectomies are performed for non-malignant diseases ${ }^{2}$. Indications for hysterectomy include Leiomyomas, endometriosis, uterovaginal (UV) prolapse (pelvic relaxation), pelvic inflammatory disease (PID), endometrial hyperplasia, dysfunctional/abnormal uterine bleeding (DUB), menorrhagia, dysmenorrhoea or pelvic pain associated with significant pelvic disease, intractable postpartum haemorrhage, ruptured tubo-ovarian abscesses, endometrial hyperplasia with atypia and malignancies such as cervical intraepithelial neoplasia or invasive Disease $\mathrm{B}^{3,4}$. Types of hysterectomy are;

1. Abdominal Hysterectomy.

2. Vaginal Hysterectomy. 
Abdominal Hysterectomy remains the predominant method of uterine removal. This route is used for malignancies, bulky uteri or when there are adhesions and removal of uterus is not possible thorugh vaginal Hysterectomy. Currently it exceeds Vaginal Hysterectomy by a ration of 1:1 to 6:1 across North America ${ }^{5}$.

Hysterectomy is reasonably safe, common and routine surgical procedure which rarely leads to peri-operative death $^{2}$. Overall mortality rates for Abdominal Hysterectomy or Vaginal Hysterectomy are $0.1-0.2 \%{ }^{6}$. It is not associated with long term risk of death ${ }^{2}$. Minor complications indluding postoperative infection, fever, wound haematoma, or separation occur in about $25 \%$ of patients, and major complications including blood transfusion ad injury to bowel, bladder, or ureer occur in $5 \%$ to $14 \%$ of patients ${ }^{7}$. The most serious post operative complication is Haemorrhage $(0.2-2 \%)^{8}$. About $10 \%$ patients are expected to have postoperative febrile morbidity and infection ${ }^{4}$. The bladder may be injured in $2.9 \%$ of all hysterectomies. Ureteral injury occurs in $0.7-1.8 \%$ of abdominal hysterectomy and $0-0.1 \%$ of vaginal hysterectomy ${ }^{9}$. Damage to the bowel is quite uncommon particularly with vaginal hysterectomy ${ }^{10}$. The advantage of vaginal hysterectomy over abdominal hysterectomy are shorter operative time, earlier hospital discharge, less post operative analgesia and quicker recovery ${ }^{10}$.

A study conducted in Abbottabad showed only 5\% patients of abdominal hysterectomy developed pyrexia against none in vaginal hysterectomy group. The results of this study also showed that mean hospital stay was $2.03 \pm 0.15$ days in Vaginal hysterectomy patients while it was $3.30 \pm 0.72$ days in patients undergoing abdominal hysterectomy ${ }^{10}$. A study carried out in Karachi showed febrile morbidity for both abdominal hysterectomy and vaginal hysterectomy group is $(9.67 \% \text { and } 9.61 \%)^{11}$. A study carried out in India showed the mean hospital stay in patients undergoing vaginal hysterectomy is $8.1 \pm 1.54$ days while it was $10.9 \pm 2.62$ days. Dorsay et al in his study showed duration of surgery was 30 minutes longer for abdominal hysterectomy than that of vaginal hysterectomy ${ }^{12}$. A study carried out in Karachi reported the hospital study in Vaginal hysterectomy group is $4.48 \pm 2.43$ days and in abdominal hysterectomy group is $5.73 \pm 2.36$ days $^{13}$. Another study conducted by Muller showed results of mean hospital stay in vaginal hysterectomy is $7.8 \pm 0.80$ days and in abdominal hysterectomy is $10 \pm 1.1$ days $^{14}$. The frequency of vaginal hysterectomy and abdominal hysterectomy is $11.87 \%$ and $28.57 \%$ respectively ${ }^{14}$.

\section{Material and Methods:}

Women fulfilling criteria of vaginal hysterectomy and abdominal hysterectomy reporting to Obstetrics and Gynecology Nishtar Hospital Multan were included in this study. Informed written consent was taken from each patient. Morbid Obesity (BMI $\geq 30$ ), diabetes mellitus, cardiac diseases, bronchial asthma, hypertension, pelvic Inflammatory Disease, endometriosis and pelvic Malignancies were excluded from our study.

Total 240 patients were included in this study who met inclusion and exclusion criteria after their admission in Nishtar Hospital Multan for their hysterectomy. Both procedure i.e. abdominal hysterectomy and vaginal hysterectomy whichever applicable was performed by an experienced surgeon having 5 years post fellowship experience. The mean duration of surgery, mean hospital stay and postoperative pyrexia were recorded in both groups. Data were entered and analyzed by using SPSS version 17. Descriptive statistics was used to analyze the data. Means and standard deviation were calculated for the age of patients, operating time (in minutes) and length of hospital stay (in days). Frequencies and percentages were calculated for vaginal hysterectomy, abdominal hysterectomy and post-operative complications (postoperative pyrexia).

\section{Results:}

A total of 240 subjects fulfilling the inclusion and exclusion criteria were registered in this study for abdominal hysterectomy and vaginal hysterectomy. Out of these 240 cases, 79 (32.9\%) had undergone vaginal hysterectomy and $161(67.1 \%)$ underwent through abdominal hysterectomy. Mean age of the study cases was $42.72 \pm 5.18$ years ranging from 31 years to 50 years, while age distribution indicates that $28(11.7 \%)$ of the study cases belonged to age group 31-35 years of age, 63 (26.3\%) were from age group 36-40 years, $100(41.7 \%)$ were from age group 41-45 years and $49(20.4 \%)$ were from age group 46-50 years. The results of this study indicated that the ages of most of the study cases i. e. 100 (41.7\%), were between $41-45$ years of age and $26.3 \%$ belonged to age group of 36-40 years of age. Mean parity of the study cases was $5.67 \pm 2.08$ (minimum para 1 and maximum para 10). Parity distribution shows that $9(3.8 \%)$ had parity $1-2$, while $62(25.8 \%)$ were from parity group 3-4, $97(40.4 \%)$ were from para 5-6 and parity groups 7-8 and 9-10 had 36 (15\%) in each group. Maximum number of study cases i. e. 159 (66.25\%) were having parity between 3-6. Pyrexia was seen in 90 
$(37.5 \%)$ of the study cases, of these $27(30 \%)$ had undergone through vaginal hysterectomy and $63(70 \%)$ underwent abdominal hysterectomy. Minimum duration of the surgery was 45 minutes and maximum duration of the surgery was 90 minutes, mean duration of the surgery was $69.75 \pm 14.92$ minutes. Minimum Hospital stay was 3 days ranging to maximum hospital stay being 10 days, mean hospital stay in these study cases was observed to be $4.42 \pm 1.65$ days.

\section{Discussion;}

Hysterectomy is the surgical removal of all or part of uterus and it is one of the most commonly performed major operations after caesarean sections. In USA, approximately sixty thousand hysterectomies are performed every year. Almost $20 \%$ ladies, before the age of 60 , undergo this procedure in United Kingdom. The findings of the recent published studies have indicated that surgeons should perform vaginal hysterectomy rather than abdominal hysterectomy whenever possible in order to cut down complications and the length of hospital stays. According to systematic evidence review by Johnson $\mathrm{N}$, women who had vaginal hysterectomy had fewer infection and high temperature after surgery compared to those who had abdominal hysterectomies. Dicker and his associates in their study found that total abdominal hysterectomy (TAH) had 1.7 times more risk of complications than those of vaginal hysterectomies. This study was planned to determine mean duration of surgery, mean duration of hospital stay and post-operative pyrexia in vaginal route and abdominal route of hysterectomies in the women with uterovaginal prolapse (UV-prolapse), uterus less than 12 week size and with failed medical treatment in dysfunctional uterine bleeding (DUB). In this study we registered a total of 240 women who underwent hysterectomy, of these $79(32.9 \%)$ have undergone vaginal hysterectomy and 161 (67.1\%) underwent through abdominal hysterectomy. Mean parity of the study cases was $5.67 \pm 2.08$ (minimum para 1 and maximum para 10). Parity distribution shows that $9(3.8 \%)$ had parity $1-2$, while $62(25.8 \%)$ were from parity group 3-4, $97(40.4 \%)$ were from para 5-6 and parity groups 7-8 and 9-10 had 36 (15\%) in each group. Maximum number of study cases i. e. 159 (66.25\%) were having parity between 3-6. Postoperative pyrexia was seen in $90(37.5 \%)$ of the study cases, of these $27(30 \%)$ had undergone through vaginal hysterectomy and $63(70 \%)$ underwent abdominal hysterectomy. These results are similar to those of reported by Ikram M ${ }^{15}$ who reported $20 \%$ postoperative pyrexia in patients with vaginal hysterectomy and $42.8 \%$ in case of abdominal hysterectomies which is lower than our results. Dawood et al ${ }^{10}$ reported quite lower rates of pyrexia in their study as only $5 \%$ pyrexia was seen in abdominal hysterectomy patients while did not observe any in those of undergoing vaginal hysterectomies. Minimum duration of the surgery was 45 minutes and maximum duration of the surgery was 90 minutes, mean duration of the surgery was $69.75 \pm 14.92$ minutes. Mean duration of surgery in vaginal hysterectomy was $68.92 \pm 15.47$ minutes while $70.16 \pm 14.67$ minutes in case of abdominal hysterectomy, these results are in compliance with the results of other studies published from Pakistan. Our results are comparable to those of Dawood NS et al ${ }^{10}$ who have reported $73.20 \pm 20.35$ minutes in case of vaginal hysterectomies. Iftikhar $R$ et al reported duration of surgery as $75 \pm 5$ minutes in case of vaginal hysterectomy, these results may show slightly higher mean duration of surgery but still close to our findings ${ }^{16}$. However our results for mean duration of surgery for abdominal hysterectomies are shorter (70.16 \pm 14.67 minutes) than those of Dawood NS et al and Iftikhar R i. e. $85.88 \pm 19.44$ and $90 \pm 5$ minutes respectively. Hysterectomy procedures are commonly employed in gynecological settings all over the world and any related potential saving would manifest remarkably in terms of cost effectiveness. Shorter hospital stays is always desirable for both hospital authorities as well as for the patients undergoing this procedure by vaginal hysterectomy. This results in lower national investments and have lower impact on national economies in terms of being cost effective. Significant reductions in national healthcare investments can be achieved if the vaginal hysterectomy predominates along with that of earlier hospital discharges, quick rates of recovery hence ultimately leading towards improved postoperative quality of life. This will also help to maintain better patient satisfaction in our society. In our study, minimum hospital stay was 3 days ranging to maximum hospital stay being 10 days, mean hospital stay in these study cases was observed to be $4.42 \pm 1.65$ days. Mean hospital stay in patients with vaginal hysterectomy was $3.77 \pm 0.47$ days while it was $4.61 \pm 1.96$ days in case of abdominal hysterectomy. Dawood NS et al reported $2.03 \pm 0.15$ days in case of vaginal hysterectomy while $3.30 \pm 0.72$ days in case of abdominal hysterectomies, these are shorter hospital stays as compared to our study results however mean hospital stay was significantly shorter $(<0.05)$ in case of vaginal hysterectomies compared with those of abdominal hysterectomies ${ }^{10}$. Similarly longer hospital stays in patients with abdominal hysterectomies have been reported by Ikram $\mathrm{M}^{15}$ who reported longer hospital stay due to postoperative wound infection. He even reported that $17.1 \%$ of the study cases undergoing through abdominal hysterectomy had to stay in hospital for more than 12 days due to infection in their wounds while none such case was reported in case of vaginal hysterectomies. The results of this current study as well as other studies conducted in Pakistan and available from other countries have found that vaginal route of hysterectomy provides faster operating technique 
compared with that of abdominal hysterectomy, even results in significantly shorter hospital stays, also associated with lesser number of peri-operative and postoperative complication rates. Our results remained in accordance with those of many studies conducted at national level.

\section{Conclusion}

Vaginal route of hysterectomy is associated with lesser postoperative complications in terms short duration surgery, significantly short hospital stays and lower rates of postoperative pyrexia than that of abdominal route of hysterectomy. Vaginal route of hysterectomy, in terms of short hospital stays, provides less burden on hospital authorities as well as being cost effective both for national economies and for patient itself. Short hospital stays ultimately lead towards better quality of life in terms of physical aspects, pain, functional capacity and less chances for nosocomial infections.

\section{References:}

1. DeFrances CJ, Hall MJ. 2005 National Hospital Discharge Survey. Adv Data 2007;(385):1-19.

2. Edozien LC. Hysterectomy for benign conditions. Br Med J 2005;330(7506):1457-8.

3. Koumans EH, Sternberg M, Bruce C, McQuillan G, Kendrick J, Sutton M, et al. The prevalence of bacterial vaginosis. In; UnMerrill RM. Hysterectomy surveillance in the United States, 1997 through 2005. Med Sci Moni 2008;14(1):CR24-CR31.

4. Saha R, Sharma M, Padhye S, Karki U, Pandey S, Thapa J. Hysterectomy: an analysis of perioperative and post operative complications. Khatmandu Univ Med J 2003;1:124-27.

5. Kovac SR. Transvaginal hysterectomy: rational and surgical approach. Obst Gynecol 2004:103:1321.

6. Harris WJ. Early complications of abdominal and vaginal hysterectomy. Obstet Gynecol Surv 1995;50:795-805.

7. Dutton S, Hirst A, McPherson K, Nicholson T, Maresh MA. UK Multicentre retrospective cohort study comparing hysterectomy and uterine artery embolisation for the treatment of symptomatic uterine fibroids (HOPEFUL Study): main results on medium-term safety and efficacy. Br JJ Obstet Gynecol 2007;114(11):1340-51.

8. McCracken G, Hunter D, Morgan D, Price JH. Comparison of laparoscopic-assisted vaginal hysterectomy, total abdominal hysterectomy and vaginal hysterectomy. Ulster Med J 20061;75(1):54-8.

9. Wu HH, Yang PY, Yeh GP, Chou PH, Hsu JC, Lin KC. The detection of ureteral injuries after hysterectomy. J Minim Invasive Gynecol 2006;13:403-8.

10. Dawood NS, Mahmood R, Haseen N. Comparison of vaginal and abdominal hysterectomy: per and post operative outcome. J Ayub Med Coll Abbottabad 2009;21(4):116-9.

11. Iftikhar R. Vaginal hysterectomy is superior than abdominal hysterectomy. J Surg Pak 2008;13:55-8.

12. Shanthini NF, Poomalar GK, Jayasaree M, Bupathy A. Evaluation of complications of abdominal and vaginal hysterectomy. Int J Reprod Contracept Obstet Gynecol 2012;1(1):7-11.

13. Tabasum R, Hasnny SF, Sabbar S, Hasnain F, Hanif F, Hanifa R. Morbidity and postoperative recovery of hysterectomy cases. J Surg Pak 2010;15(2):73-77.

14. Muller A, Thiel FC, Renner SP, Winkler M, Haberle L, Beckmann MW. Hysterectomy- a comparison of approaches. Dtsch Aratebl Int 2010;107(20):353-9.

15. Ikram M, Saeed Z, Saeed R, Saeed M. Abdominal versus vaginal hysterectomy. Professional Med J 2008;15(4):486-91.

16. Iftikhar R. Vaginal Hysterectomy is superior than abdominal hysterectomy. J Surg Pak 2008;13(2):55-58 